\title{
DETERMINAÇÃO DO ÍNDICE DE ESTADO TRÓFICO DO CÓRREGO LISO PERTENCENTE À MICROBACIA DO RIO SÃO JOÃO, MINAS GERAIS E COMPARAÇÃO COM PROTOCOLO DE AVALIAÇÃO RÁPIDA
}

\author{
Eline dos Santos Oliveira ${ }^{1}$
}

Thainá Desiree Franco dos Reis ${ }^{2}$

Odila Rigolin de Sá ${ }^{3}$

\begin{abstract}
RESUMO
A água é de fundamental importância para a vida das espécies, pensando nisso é importante lembrar que quando aumentam as atividades antrópicas aumentam também as fontes de poluição, tais como o lançamento de efluentes não tratados alterando negativamente a qualidade da água. Sendo assim, sua conservação é essencial e para isso é primordial seu monitoramento. Assim objetivou-se neste trabalho calcular o Índice de Estado Trófico e comparar com o Protocolo de Avaliação Rápida (PAR's) segundo Callisto et al. (2002) no Córrego Liso, São Sebastião do Paraíso, Minas Gerais. A coleta e aplicação do protocolo foi realizada no mês de Junho em cinco pontos de amostragem ao longo de um gradiente longitudinal. Para a classificação do PAR's apenas o ponto 1 (montante) foi considerado "natural", o restante foi classificado com "impactado", o índice de estado trófico calculado de todos os pontos foi considerado como hipereutrófico, o que reforça o grau de gravidade em que o córrego se encontra. Apesar do extenso trabalho de monitoramento realizado pelo IGAM, medidas mais rápidas precisam ser tomadas, pois a captação de água da cidade ocorre no Rio Santana que recebe as águas do Córrego Liso, tendo de passar por uma estação de tratamento pelo método convencional avançado, o que torna a água mais cara para a população.
\end{abstract}

PALAVRAS-CHAVE: Habitats, Índice de Estado Trófico, Ambientes lóticos.

\section{DETERMINATION OF TROPHIC STATE INDEX STREAM LISO BELONGING TO SÃO JOÃO RIVER WATERSHED, MINAS GERAIS AND COMPARISON WITH RAPID ASSESSMENT PROTOCOL}

\footnotetext{
${ }^{1}$ Graduanda em Ciências Biológicas, FESP/UEMG. E-mail: eline.oliveira@hotmail.com.br ${ }^{2}$ Graduanda em Ciências Biológicas, FESP/UEMG. E-mail: thainadfr@yahoo.com.br ${ }^{3} \mathrm{Dr}^{\mathrm{a}}$ em Ecologia e Recursos Naturais, coordenadora e professora do curso de Ciências Biológicas, FESP/UEMG. E-mail: odilarigolin@yahoo.com.br
} 


\begin{abstract}
Water is fundamental for life species, thinking it is important to remember that when increase anthropogenic activities also increase the sources of pollution such as the release of treated effluent does not negatively altering water quality. Thus conservation is essential and this is your primary monitor. Thus the aim of this work to calculate the Trophic State Index and compare with the Rapid Assessment Protocol (PAR's) seconds Callisto et al. (2002) in Liso Stream, São Sebastião do Paraíso, Minas Gerais. The collection and application of the Protocol was held in the month of Junho in five sampling points along a longitudinal gradient. For the classification of PAR's just the point 1 (amount) was considered 'natural', the rest was rated 'impacted' calculated, the Trophic State Index of all points was regarded as hypereutrophic, which reinforces the seriousness in the stream is. Despite the extensive work done by monitoring IGAM, faster measures need to be taken, for the capture of city water that occurs in Santana River receives the waters of Liso Stream, having to go through a treatment plant by conventional advanced method, the which makes it the most expensive water for the population.
\end{abstract}

KEY-WORDS: Habitats, Trophic State Index, Lotic Environments.

\title{
DETERMINACIÓN DEL ÍNDICE DE ESTADO TRÓFICO DEL ARROYO LISO PERTENECIENTE A LA CUENCA DEL RÍO SÃO JOÃO, MINAS GERAIS Y COMPARACIÓN CON EL PROTOCOLO DE EVALUACIÓN RÁPIDA
}

\section{RESUMEN}

El agua tiene fundamental importancia para la vida de las especies, pensando em eso, es importante recordar que cuando se aumentan las actividades antropogénicas también aumentan las fuentes de contaminación, tales como la liberación de efluentes no tratados que cambian negativamente la calidad del agua. Por lo tanto, su conservación es esencial y el monitoreo primordial. Así, este trabajo tuvo como objetivo calcular el Índice de Estado Trófico y comparar con el Protocolo de Evaluación Rápida (PER's) según Callisto et al. (2002) en el Arroyo Liso, São Sebastião do Paraíso, Minas Gerais. La recolecta y aplicación del Protocolo se realizó en junio en cinco puntos de muestreo a lo largo de un gradiente longitudinal. Para la clasificación del PER sólo el punto 1 (montante) se consideró "natural", el resto fue calificado como 'impactado', el índice de estado trófico calculado para todos los puntos se consideró como hipereutrófico, lo que refuerza la grave situación que el río se encuentra. A pesar del extenso labor de monitoreo realizado por el IGAM, medidas más rápidas se deben tomar, pues la captura de agua de la ciudad ocurre en el Río Santana que recibe las aguas del Arroyo Liso, pasando por una planta de tratamiento por el método convencional avanzado, lo que hace el agua más cara para la población.

PALABRAS CLAVE: Hábitat, Índice de Estado Trófico, Ambientes Lóticos.

\section{INTRODUÇÃO}

A água constitui um dos compostos de maior distribuição e importância na crosta terrestre. Sua importância para a vida está no fato de que nenhum processo metabólico ocorre sem a sua ação direta ou indireta. Foram suas propriedades 
anômalas, comparando com outros compostos, que possibilitaram o surgimento e a manutenção da vida na Terra (ESTEVES, 1998).

Pode-se observar que os ecossistemas aquáticos, nas últimas décadas, têm sido alterados de maneira significativa em função de múltiplos impactos ambientais, oriundos de diversas atividades, tais como: mineração, construção de barragens e represas, reutilização e desvio do curso natural de rios, lançamento de efluentes domésticos e industriais não tratados, desmatamento e uso inadequado do solo em regiões ripárias e planícies de inundação, super exploração de recursos pesqueiros e introdução de espécies exóticas. Como consequência destas atividades, tem-se observado uma expressiva queda da qualidade da água e perda de biodiversidade aquática em função da desestruturação do ambiente físico e alteração da dinâmica natural das comunidades biológicas (GOULART; CALLISTO, 2003).

Para conceituar a qualidade de uma determinada água, deve-se ponderar a relação das condições naturais do uso e ocupação do solo na bacia hidrográfica. A qualidade das águas depende das condições geológicas, geomorfológicas e da cobertura vegetal da bacia de drenagem, além do comportamento dos ecossistemas terrestres e aquáticos e das ações do homem (TUCCI, 2007).

A implementação de estudos de avaliação trófica em sistemas aquáticos tem como relevância a detecção e predição dos seus processos de eutrofização e busca de propostas de soluções que viabilizem o aumento da vida útil desses ecossistemas (TUNDISI, 1999).

A avaliação do grau de eutrofização em lagos e reservatórios e feita tradicional mente em diversos países. A tradição de limnologia no estudo de lagos, seu uso para abastecimento eo aparecimento mais "precoce" de efeitos do enriquecimento de corpos de agua lênticos são algumas das razões para a priorização dos estudos sobre eutrofização nesses ambientes, em relação aos ambientes lóticos (LAMPARELLI, 2004).

A principal fonte de contaminação é o despejo de material tóxico proveniente das atividades agroindustriais e industriais, responsáveis por $90 \%$ do consumo das 
águas, devolvendo-as à natureza completamente contaminadas. No caso das indústrias, o agravante está na falta de controle ambiental quanto a geração, tratamento e destinação final de resíduos gerados no processo produtivo, normalmente acumulados nas margens de cursos de água. (DEFENSORIA DAS ÁGUAS, 2004).

A água é o elemento mais importante para da indústria do couro, pois ela é utilizada na maioria das operações realizadas no curtume. De acordo com Câmara e Gonçalves Filho (2007), a água é utilizada como solvente nos banhos de tratamento e nas lavagens das peles. Nessas duas etapas, a água entra limpa e sai acrescida de resíduos orgânicos e de produtos químicos, gerando uma mistura de efluentes com alto poder de contaminação e degradação do meio ambiente.

Os Protocolos de Avaliação Rápida de Rios (PARs), nascidos de estudos liderados pela Environmental Protection Agency (EPA), durante a década de 1980, em conjunto com agências de monitoramento de águas superficiais, são utilizados para caracterizar o rio qualitativamente. No Brasil o PAR's foi adaptado por Callisto et al. (2002).

É estabelecido, em princípio, um limite considerado normal baseado em locais minimamente perturbados sendo estes tomados como "referência" (Plafkinet al., 1989). Eles constituem-se em documentos de referência que reúnem procedimentos metodológicos aplicáveis à avaliação rápida, qualitativa e semi-quantitativa, de um conjunto de variáveis representativas dos principais componentes e fatores geomórficos e sedimentológicos, que condicionam e controlam os processos e funções ecológicas dos sistemas fluviais (Rodrigues e Castro, 2008; Rodrigues et al., 2008).

As exigências de cuidado com a água que os curtumes descartam são dirigidas através de regulamentação do Conselho Nacional de Meio Ambiente (CONAMA), em nível federal, e dos órgãos responsáveis pelo meio ambiente, em nível estadual e municipal. São essas leis que determinam a classificação dos tipos das águas existentes, e dos critérios de qualidade do efluente na hora de seu descarte na rede pública de esgoto ou no leito de algum rio (OLIVEIRA, 2008). 
Objetivou-se com este trabalho estimar o Índice de Estado Trófico do Córrego Liso pertencente à micro bacia do Rio São João, localizada na bacia hidrográfica do médio Rio Grande, e comparar com o Protocolo de Avaliação Rápida (Callisto, 2002).

\section{MATERIAIS E MÉTODOS}

\section{1 Área de estudo}

O presente trabalho teve por finalidade avaliar a qualidade da água através de análises químicas, para calcular o índice de estado trófico e realizar o protocolo de avaliação rápida em cinco pontos de amostragem. O córrego Liso é considerado de grande importância visto que captação da água da cidade de São Sebastião do Paraíso ocorre no Rio Santana que recebe as águas do Córrego Liso. 
Figura 1: Área de abrangência da sub-bacia estudada. Esta sub-bacia está localizada na bacia hidrográfica do médio Rio Grande - MG.

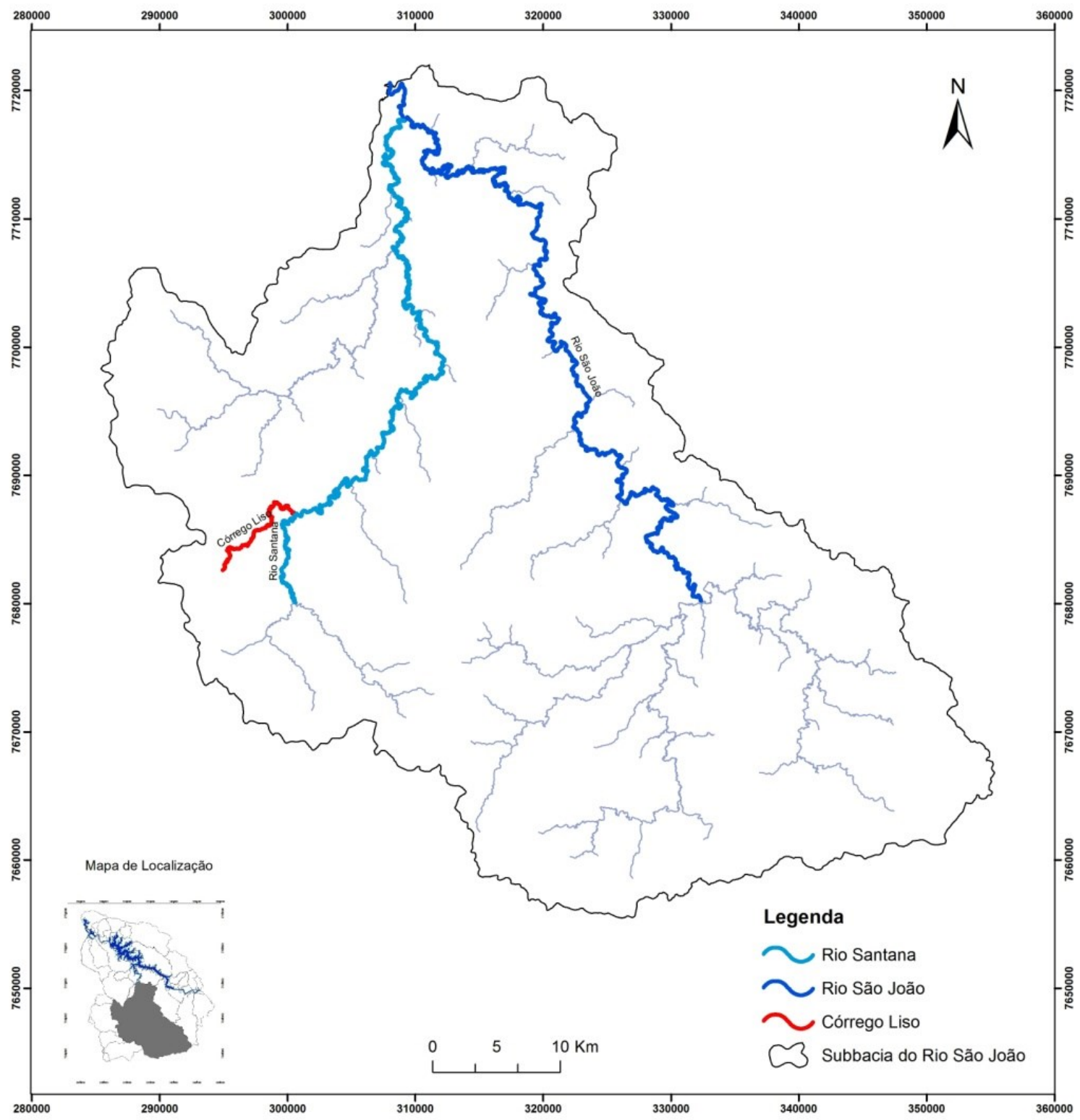

\subsection{Amostragem}

A coleta foi realizada no mês de Junho de 2014, com cinco amostragens de água em triplicata. Os pontos foram escolhidos ao longo do gradiente longitudinal e 
classificados da seguinte maneira: sendo o ponto 1 montante, pontos 2, 3, 4 intermediários ao longo do gradiente longitudinal e o ponto 5 jusante.

Amostras de água foram obtidas abaixo da lâmina d'água com garrafas de polietileno de $1000 \mathrm{ml}$ para a determinação das variáveis. O acondicionamento e conservação das amostras foram realizados conforme métodos especificados em "Standard Methods for the Examination of Water and Wastewater" (APHA, 1998).

\subsection{Análises químicas}

As análises de clorofila a foram realizadas segundo a metodologia proposta por Lorenzen (1967), e fósforo total segundo de Strickland \& Parsons (1960).

\section{4 Índice de Estado Trófico}

O IET foi calculado segundo propostas de Carlson (1977) modificado por Toledo (1990) e Lamparelli (2004). O IET geral que calcula a média aritmética simples dos índices relativos ao fósforo total e a clorofila a:

$$
\text { IET = [ IET ( PT ) + IET ( CL) ] } 2
$$

Os limites estabelecidos para as diferentes classes de trofia geral são: ultraoligotrófico (IET $\leq 47$ ); oligotrófico (47 < IET $\leq 52)$; mesotrófico (52 < IET $\leq 59$ ); eutrófico (59 < IET $\leq 63)$; supereutrófico $(63<$ IET $\leq 67)$ e hipereutrófico (IET> 67).

\subsection{Protocolo de Avaliação Rápida (PAR's)}

Segundo Callisto et al. (2002), os protocolos são instrumentos que visam avaliar a estrutura e o funcionamento dos ecossistemas aquáticos, no sentido de contribuir para o manejo e conservação destes ambientes baseados em parâmetros de fácil entendimento e utilização simplificada. O protocolo utilizado possui 22 parâmetros, e é dividido em duas partes, ou seja, os 10 primeiros parâmetros são aqueles que avaliam as condições dos trechos que possam ter sofrido alterações 
pelas atividades antrópicas, eles recebem a pontuação 0 à 4 . Os 12 parâmetros restantes avaliam as condições naturais/ecológicas da área e podem ser pontuados de 0 à 5 . Os trechos são classificados da seguinte maneira; de 0 a 40 pontos: impactado, 41 a 61 pontos: alterado e de 61 a 100 pontos: natural.

\section{RESULTADOS E DISCUSSÃO}

De acordo com Zalewski \& Robarts (2003), uma avaliação dotada de caráter isolado, não contempla, de maneira global, a real situação do meio. É necessário que abordagens interativas, como as que englobam aspectos geomórficos, sedimentológicos, ecológicos, físico-químicos e biológicos das águas, sejam adotadas, afim de que cada um destes se completem mutuamente, disponibilizando informações mais sistêmica sobre a qualidade dos recursos hídricos (Karr \& Chu, 1999; Barbour \& Stribling, 1991).

Os resultados obtidos pelo PAR's mostrou que o P1 (montante) foi o único ponto considerado como "natural", e os pontos seguintes foram classificados como "impactados", ou seja, totalmente alterados pela ação antrópica deixando com que percam totalmente sua característica natural. De acordo com os resultados obtidos pelo estudo de Souza et al. (2014), que também utilizou o PAR's como ferramenta para avaliar a qualidade do córrego liso, mostrou que em seis pontos estudados também foram enquadrados como "impactados". 


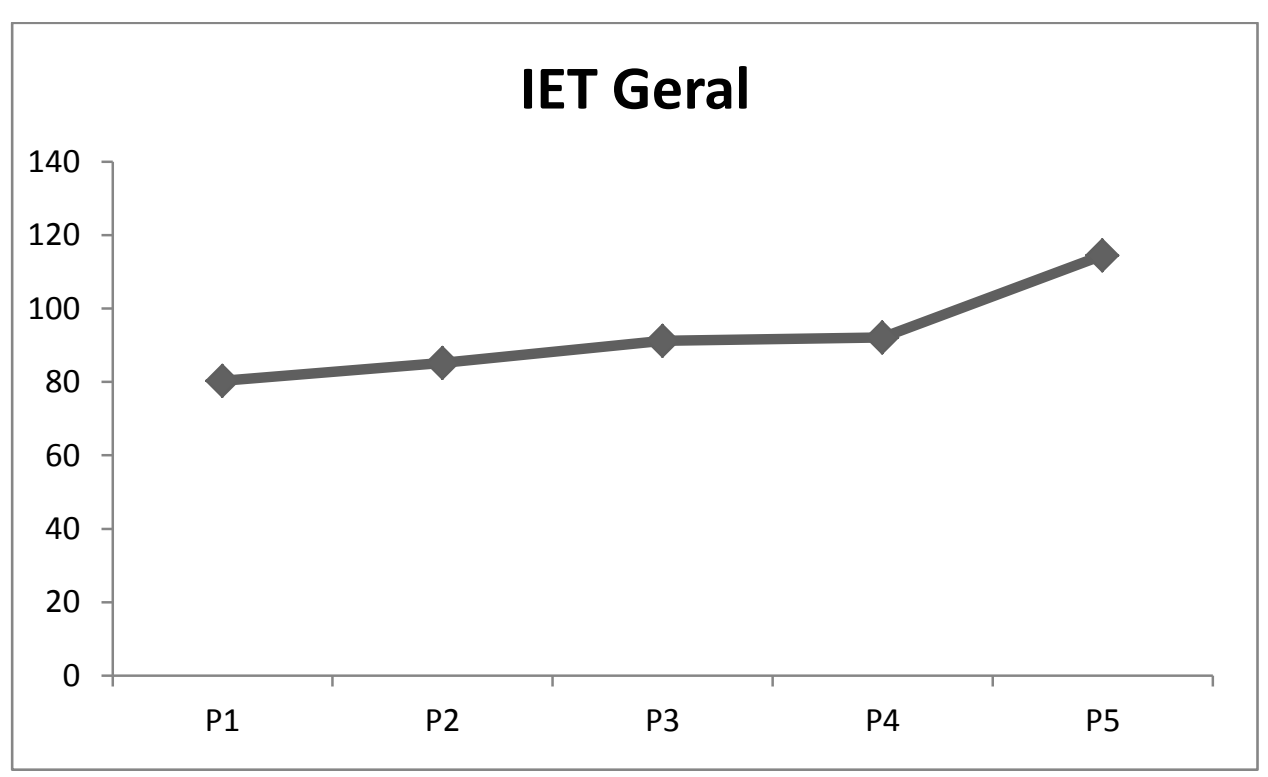

Gráfico 1: Resultados do Índice de Estado Trófico geral calculado a partir da medida dos resultados do IET da clorofila a calculado em $\mathrm{mg} \mathrm{L-1}$ e IET do fósforo total calculado em mg L-1.

O IET geral calculado de todos os pontos foi considerado como hipereutrófico, já que todos apresentaram IET> 67, como mostra a gráfico 1. Apesar do P1 ter sido o único considerado como "natural" e também apresentar o menor resultado para o IET entre os outros pontos, o mesmo apresenta um IET altíssimo, o que reforça a utilização benéfica em conjunto de diversas metodologias para o estudo de um recurso hídrico.

A elevação no valor do IET nos últimos três pontos pode ser justificada pelo fato de que anteriormente ao $\mathrm{P} 3$, existe uma indústria de curtume que provavelmente lança uma grande quantidade de efluentes, que contem vários químicos em sua composição referentes aos processos de lavagem, e geralmente é o que causa alteração na cor e no cheiro da água do córrego Liso. 
Tabela 1: Média dos resultados das análises de fósforo total e clorofila a.

\begin{tabular}{c|c|c}
\hline & $\begin{array}{c}\text { Fósforo Total } \\
\text { mg L-1 }\end{array}$ & $\begin{array}{c}\text { Clorofila } \mathbf{a} \\
\text { mg L-1 }^{-1}\end{array}$ \\
\hline P1 & 0,006 & 11,49 \\
\hline P2 & 0,085 & 17,08 \\
\hline P3 & 0,073 & 12,29 \\
\hline P4 & 0,095 & 15,03 \\
\hline P5 & 0,203 & 30,74 \\
\hline
\end{tabular}

O resultado da análise de fósforo total (Tabela 1) do P5 mostrou-se um pouco superior aos resultados apresentados pelos três últimos Relatórios de Águas Superficiais realizados pelo IGAM no ano de 2013, este parâmetro já não atendia e continua não atendendo os limites determinados para água de classe II de acordo com a Resolução CONAMA 357/2005.

O menor número constatado para o IET geral no presente estudo foi de 80,34 (hipereutrófico) tendo um aumento significante em relação aos resultados apresentados pelo IGAM (2012), onde o Córrego Liso já se classificava como hipereutrófico, tendo como valor de IET de 67,87. Isso nos demostra a crescente demanda incessante de lançamento de efluentes industriais não tratados em todos os trechos do Córrego Liso, que ao passar dos anos está prejudicando cada vez mais a saúde do córrego.

\section{CONCLUSÃO}

O presente estudo mostrou que a utilização de metodologias diferentes para o diagnóstico de um recurso hídrico é de suma importância. A aplicação do Protocolo de Avaliação Rápida foi complementar ao cálculo do Índice de Estado Trófico, visto que, a visão holística dos profissionais que trabalham com o meio ambiente é uma 
característica valiosa e precisa ser exercida para a detecção de fatores que possam estar interferindo no meio.

Existem projetos para a inserção de uma futura Estação de Tratamento de Esgoto (E.T.E.), pois comumente o Córrego Liso recebe efluentes de atividades de industriais de vários tipos e esgoto doméstico. Apesar do amplo trabalho de monitoramento realizado pelo IGAM, medidas mais rápidas precisam ser tomadas, pois a captação de água da cidade ocorre no Rio Santana que recebe as águas do Córrego Liso, trazendo assim uma grande chance de contaminação da população e de animais que usufruem dessa água.

\section{REFERÊNCIAS}

APHA (1998). Standard Methods for the Examination of Water and Wastewater, 20th edition.

BARBOUR, M.T., \& STRIBLING, J.B.. 1991. Use of habitat assessment in evaluating the biological integrity of stream communities. In George Gibson, editor. Biological criteria: Research and regulation, proceedings of a symposium, 12-13 December 1990, Arlington, Virginia. Office of Water, U.S. Environmental Protection Agency, Washington, D.C. EPA-440-5-91-005.

CALLISTO, M.; FERREIRA, W. R.; MORENO P.; GOULART M.; PETRUCIO M. Aplicação de um protocolo de avaliação rápida da diversidade de habitats em atividade de ensino e pesquisa (MG-RJ). Acta Limnologica Brasiliensia. v. 14, n. 1, p. 91-98, 2002.

CÂMARA, R. P. de B.; GONÇALVES FILHO, E. V. Análise dos custos ambientais da indústria de couro sob a ótica da eco-eficiência. Custos e @gronegócio on-line, n.1, p. 87-100, Jan./Jun.2007. Disponível em: http://www.custoseagronegocioonline.com.br/numero1v3/custos\%20ambientais.pdf. Acessado em: 01 set. 2014.

CARLSON, R. E. 1977. A trophic state index for lakes.Limnology and Oceanography. 22(2): 361369.

DEFENSORIA DAS ÁGUAS. O Estado Real das Águas no Brasil - 2003/2004. 2004. Disponível em: <http://www.daerp.ribeiraopreto.sp.gov.br>. Acessado em: 09 de ago. de 2014.

ESTEVES,F.A. Fundamentos de limnologia. Rio de Janeiro: Interciência, 1998, 575p.

GOULART, M. D.; CALLISTO, M. Bioindicadores de Qualidade de Água como ferramenta em estudos de impacto ambiental. Revista FAPAM, v.2, p.78-85, 2003. Disponível em: <http://www.revistas2.uepg.br/index.php/tp/article/view/1876/2427>. Acesso em: 05 set. 2014.

IGAM. Instituto Mineiro de Gestão das Águas. Relatório Trimestral. Belo Horizonte, $1^{\circ}$ trimestre de 2012. 
KARR, J.R., \& CHU, E.W. 1999. Restoring life in running waters: Better biological monitoring.Island Press, Washington, D.C.

LAMPARELLI, Marta C. Grau de Trofia em Corpos D’Água do Estado de São Paulo: Avaliação dos Métodos de Monitoramento. 238 p. Tese (Doutorado)- Instituto de Biociências, Universidade de São Paulo, São Paulo, 2004.

Lorenzen C. J., 1967, Determination of chlorophyll and phaeopigments: spectrophotometric equations, Limnol. Oceanogr., 12

OLIVEIRA, G. J. de. Jeans: a alquimia da Moda. Vitória: Edição independente, 2008.

PLAFKIN, J. L.; BARBOUR, M. T.; PORTER, K. D.; GROSS, S. K.; HUGHES, R. M. Rapid bio assessment protocols for use in streams and rivers: benthic macroinvertebrates and fish. Washington: EPA 440-4-89-001, 1989. 339 p.

Resolução $\mathbf{n}^{\circ}$. 357, de 17 de março de 2005. Dispõe sobre a classificação dos corpos de água e diretrizes ambientais para o seu enquadramento, bem como estabelece as condições e padrões de lançamento de efluentes, e dá outras providências. Conselho Nacional do Meio Ambiente (CONAMA). Publicada no Diário Oficial da União, nº. 053, de 18/03/2005, p. 58-63.

RODRIGUES, A. S. L. Adequação de um protocolo de avaliação rápida para o monitoramento e avaliação ambiental de cursos d'água inseridos em campos rupestres. 2008. 146 f. (Mestrado em Ciências Naturais) - Programa de Pós Graduação em Evolução Crustal e Recursos Naturais, Departamento de Geologia, Universidade Federal de Ouro Preto, Ouro Preto, 2008.

RODRIGUES, A. S. L.; CASTRO, P. T. A. Protocolos de avaliação rápida: instrumentos complementares no monitoramento dos recursos hídricos. Revista Brasileira de Recursos Hídricos, v. 13, n. 1, 161-170, 2008.

SOUZA, A. C; RIGOLIN-SÁ, O. Comparação entre o índice de estado trófico (IQA) com o protocolo de avaliação rápida de habitats no Córrego Liso no município de São Sebastião do Paraíso, Minas Gerais. 2014. Artigo de Trabalho de Conclusão de Curso (Bacharel em Engenharia Ambiental) - Fundação de Ensino Superior de Passos. Faculdade de Engenharia de Passos. Passos, MG.

STRICKLAND, J. D. \& PARSONS, T. R.A manual of seawater analysis.Bull. Fins. Res. Bel. Can., v.125, p.1-185. 1960.

TOLEDO Jr., A. P. Informe preliminar sobre os estudos para a obtenção de um índice para avaliação do estado trófico de reservatórios de regiões quentes tropicais. São Paulo: CETESB, 1990. 12p. (Relatório Interno Cetesb).

TUCCI, C. E. M. Mudanças climáticas e impactos sobre recursos hídricos no Brasil. Ciência \& Ambiente, Santa Maria, RS, n. 34, p. 137-156, 2007.

TUNDISI, J. G. Limnologia no século XXI: perspectivas e desafios. Instituto Internacional de Limnologia, São Carlos, SP, 24 p., 1999.

ZALEWSKI M, ROBARTS R. 2003. Ecohydrology-a new paradigm for integrated water resources management. SIL News 40: 1-5 